\title{
Principles of bar induction and continuity on Baire space ${ }^{1}$
}

\author{
TATSUJI KAWAI
}

\begin{abstract}
Brouwer-operations, also known as inductively defined neighbourhood functions, provide a good notion of continuity on Baire space which naturally extends that of uniform continuity on Cantor space. In this paper, we introduce a continuity principle for Baire space which says that every pointwise continuous function from Baire space to the set of natural numbers is induced by a Brouweroperation.

Working in Bishop constructive mathematics, we show that the above principle is equivalent to a version of bar induction whose strength is between that of the monotone bar induction and the decidable bar induction. We also show that the monotone bar induction and the decidable bar induction can be characterised by similar principles of continuity.

Moreover, we show that the $\Pi_{1}^{0}$ bar induction in general implies LLPO (the lesser limited principle of omniscience). This, together with the fact that the $\Sigma_{1}^{0}$ bar induction implies LPO (the limited principle of omniscience), shows that an intuitionistically acceptable form of bar induction requires the bar to be monotone.
\end{abstract}

2010 Mathematics Subject Classification 03F60, 03F55 (primary); 03F03 (secondary)

Keywords: Constructive mathematics, Bar induction, Continuity principle, Brouwer operation, Baire space

\section{Introduction}

The uniform continuity principle (UC) is the following statement:

UC Every pointwise continuous function $F:\{0,1\}^{\mathbb{N}} \rightarrow \mathbb{N}$ is uniformly continuous. In classical mathematics, the above statement is true because Cantor space $\{0,1\}^{\mathbb{N}}$ is topologically compact. This is not the case in Bishop constructive mathematics [4]. In fact, UC implies the decidable version of Brouwer's fan theorem to which there is

\footnotetext{
${ }^{1}$ This paper is included in the Proceedings of the Fifth Workshop on Formal Topology, Institut Mittag-Leffler, June 2015 (editors Thierry Coquand, Maria Emilia Maietti and Erik Palmgren).
} 
a well-known recursive counterexample (see Troelstra and van Dalen [10, Chapter 4, Section 7.6]). Here, the fan theorem is a statement saying that every bar of Cantor space is uniform (see Section 2 for terminology).

The connection between UC and the fan theorem is well studied in constructive reverse mathematics (Ishihara [6]). It is well known that the fan theorem is equivalent to compactness of Cantor space [10, Chapter 4, Section 6], and hence it implies UC. Josef Berger [2] showed that a weaker version of UC is equivalent to the decidable fan theorem (see also Remark 5.4). In another paper [3], he also introduced a variant of fan theorem, called c-FT, and showed that it is equivalent to UC.

In this paper, we establish analogous correspondence between several notions of continuity on Baire space $\mathbb{N}^{\mathbb{N}}$ and a variety of bar induction. Our focus is on the relation between various versions of bar induction and statements similar to UC, but we consider functions on Baire space instead of Cantor space and replace uniform continuity with a suitable notion of continuity on Baire space. More precisely, we consider a function from $\mathbb{N}^{\mathbb{N}}$ to $\mathbb{N}$ induced by a Brouwer-operation (Kreisel and Troelstra [9, Section 3]) to be a fundamental notion of continuity on Baire space. The notion can be considered as a natural generalisation of that of uniform continuity on Cantor space to the setting of Baire space, since it becomes equivalent to uniform continuity when restricted to Cantor space (see Proposition 3.2).

We now summarise our main contributions. First, we formulate a continuity principle for Baire space called the principle of Brouwer continuity ( $\mathrm{BC}$ ), based on the notion of Brouwer-operation. The principle BC states that every pointwise continuous function from Baire space to the set of natural numbers is induced by a Brouwer-operation. Then, we introduce a variant of bar induction, called the continuous bar induction (c-BI), and show that $\mathrm{c}-\mathrm{BI}$ is equivalent to $\mathrm{BC}$. Moreover, we characterise the other versions of bar induction, the monotone bar induction and the decidable bar induction, by a stronger and a weaker version of $\mathrm{BC}$ by varying the strength of the premise of BC. Finally, we show that the $\Pi_{1}^{0}$ bar induction (of which c-BI is an instance) in general implies the non-constructive principle LLPO (the lesser limited principle of omniscience), and thus intuitionistically unacceptable.

The relation between several versions of bar induction and continuity axioms (namely strong and weak continuity for numbers, and bar continuity) has been extensively studied by Howard and Kreisel [5] and Kreisel and Troelstra [9]. Some of their results are recalled as corollaries of our work in Section 6 (Theorem 6.1). Our main contribution is in introducing the bar induction c-BI which is equivalent to $\mathrm{BC}$ and characterising the other versions of bar induction by similar principles of continuity. In this way, the 
difference between various versions of bar induction can be understood as the difference between the notions of continuity involved in the corresponding principles of continuity.

\section{Formal system}

We work in Bishop constructive mathematics [4]. However, our work should be formalisable in a suitable extension of intuitionistic arithmetic in all finite types ( $\mathrm{HA}^{\omega}$ ), which we now briefly describe.

First, the language of $\mathrm{HA}^{\omega}$ is extended with the types of boolean $\{0,1\}$ and finite sequences $\{0,1\}^{*}$ and $\mathbb{N}^{*}$ of $\{0,1\}$ and $\mathbb{N}$ respectively, together with appropriate constructors and axioms for these types. Second, we assume the following choice axioms:

$$
\begin{aligned}
\mathbf{A C}_{01} & (\forall x \in \mathbb{N})\left(\exists \alpha \in \mathbb{N}^{\mathbb{N}}\right) A(x, \alpha) \rightarrow\left(\exists F \in\left(\mathbb{N}^{\mathbb{N}}\right)^{\mathbb{N}}\right)(\forall x \in \mathbb{N}) A(x, F(x)) \\
\mathbf{A C}_{10} ! \quad\left(\forall \alpha \in \mathbb{N}^{\mathbb{N}}\right)(\forall x \in \mathbb{N}) \neg B(\alpha, x) \vee B(\alpha, x) & \\
\rightarrow & {\left[\left(\forall \alpha \in \mathbb{N}^{\mathbb{N}}\right)(\exists ! x \in \mathbb{N}) B(\alpha, x) \rightarrow\left(\exists F \in \mathbb{N}^{\left(\mathbb{N}^{\mathbb{N}}\right)}\right)\left(\forall \alpha \in \mathbb{N}^{\mathbb{N}}\right) B(\alpha, F(\alpha))\right] }
\end{aligned}
$$

Moreover, we add a predicate symbol $K$ on $\mathbb{N}^{\mathbb{N}^{*}}$ together with the following axioms (for the notation used, see the next subsection):

$\mathrm{K} 1 \quad \lambda a \cdot x+1 \in K$

$\mathrm{K} 2 \quad[\alpha(\langle\rangle)=0 \wedge(\forall x \in \mathbb{N}) \lambda a . \alpha(\langle x\rangle * a) \in K] \rightarrow \alpha \in K$

$\mathrm{K} 3 \quad\left(\forall \alpha \in \mathbb{N}^{\mathbb{N}^{*}}\right)[A(Q, \alpha) \rightarrow Q(\alpha)] \rightarrow K \subseteq Q$

where

$A(Q, \alpha) \stackrel{\text { def }}{\Longleftrightarrow}(\exists x \in \mathbb{N})[\alpha=\lambda a \cdot x+1] \vee[\alpha(\langle\rangle)=0 \wedge(\forall x \in \mathbb{N}) \lambda a \cdot \alpha(\langle x\rangle * a) \in Q]$.

The predicate $K$ can be understood as being inductively defined by K1 and K2.

The system described above can be thought of as an extension of the intuitionistic theory of analysis IDB $_{1}$ described in Kreisel and Troelstra [9] to all finite types, together with the axiom of unique choice $\mathrm{AC}_{10}$ !. See Troelstra and van Dalen $[10,11]$ for the details of the systems $\mathrm{HA}^{\omega}$ and $\mathrm{IDB}_{1}$.

\section{Notation}

We adopt the following notation in this paper. The letters $k, n, m, x, y$ range over natural numbers $\mathbb{N}$. The letters $a, b$ range over the finite sequences $\mathbb{N}^{*}$ of natural 
numbers or the finite binary sequences $\{0,1\}^{*}$. Greek letters $\alpha, \beta, \gamma, \ldots$ range over the infinite sequences $\mathbb{N}^{\mathbb{N}}$ or $\{0,1\}^{\mathbb{N}}$. We write $|a|$ for the length of $a$ and $a * b$ for the concatenation of $a$ and $b$. We write \langle\rangle and $\langle n\rangle$ for the empty sequence and a sequence of length 1 . We write $a \preccurlyeq b$ to mean that $a$ is an initial segment of $b$. Moreover, we write $\bar{\alpha} k$ for the initial segment of $\alpha$ of length $k$, and we let $\alpha \in a$ abbreviate $\bar{\alpha}|a|=a$. We extend concatenation between finite sequences to the one between finite sequences and infinite sequences by letting $a * \alpha$ denote the sequence such that $a * \alpha \in a$ and

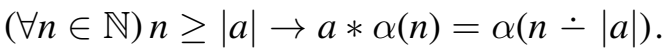

We let $A, B, C, \ldots$ range over the formulas of our system. By a predicate of type $\mathbb{T}$, we mean a formula $A$ of our system with a free variable of type $\mathbb{T}$. In this case, we write $A \subseteq \mathbb{T}$. For predicates $A, B \subseteq \mathbb{T}$, we let $A \subseteq B$ abbreviate $(\forall t \in \mathbb{T}) A(t) \rightarrow B(t)$. We sometimes write $t \in A$ for $A(t)$.

\section{Continuous bar induction}

We introduce the principle c-BI, the continuous bar induction, and argue that c-BI naturally extends the fan theorem c-FT by Berger [3].

A predicate $P \subseteq \mathbb{N}^{*}$ is a bar if

$$
\left(\forall \alpha \in \mathbb{N}^{\mathbb{N}}\right)(\exists n \in \mathbb{N}) P(\bar{\alpha} n) .
$$

A bar $P$ is a $c$-bar if there exists a function $\delta: \mathbb{N}^{*} \rightarrow \mathbb{N}$ such that

$$
\left(\forall a \in \mathbb{N}^{*}\right)\left[P(a) \leftrightarrow\left(\forall b \in \mathbb{N}^{*}\right) \delta(a)=\delta(a * b)\right] .
$$

A predicate $Q \subseteq \mathbb{N}^{*}$ is inductive if

$$
\left(\forall a \in \mathbb{N}^{*}\right)[(\forall n \in \mathbb{N}) Q(a *\langle n\rangle) \rightarrow Q(a)] .
$$

The continuous bar induction (c-BI) is the following statement:

c-BI For any c-bar $P \subseteq \mathbb{N}^{*}$ and a predicate $Q \subseteq \mathbb{N}^{*}$, if $P \subseteq Q$ and $Q$ is inductive, then $Q(\langle\rangle)$.

In the rest of this section, we relate $\mathrm{c}-\mathrm{BI}$ to the fan theorem $\mathrm{c}-\mathrm{FT}$.

We recall the standard terminology. If $P$ and $Q$ are predicates of some type $\mathbb{T}$ such that $P \subseteq Q$, we say that $P$ is detachable from $Q$ if

$$
(\forall t \in \mathbb{T}) Q(t) \rightarrow \neg P(t) \vee P(t) .
$$


A predicate $C \subseteq\{0,1\}^{*}$ is a $c$-set if there exists a detachable predicate $D \subseteq\{0,1\}^{*}$ such that

$$
\left(\forall a \in\{0,1\}^{*}\right)\left[C(a) \leftrightarrow\left(\forall b \in\{0,1\}^{*}\right) D(a * b)\right] .
$$

A predicate $P \subseteq\{0,1\}^{*}$ is a bar of the binary tree $\{0,1\}^{*}$ if

$$
\left(\forall \alpha \in\{0,1\}^{\mathbb{N}}\right)(\exists n \in \mathbb{N}) P(\bar{\alpha} n) .
$$

A bar $P \subseteq\{0,1\}^{*}$ is uniform if

$$
(\exists N \in \mathbb{N})\left(\forall \alpha \in\{0,1\}^{\mathbb{N}}\right)(\exists n \leq N) P(\bar{\alpha} n) .
$$

The principle c-FT is the following statement [3]:

c-FT Every bar $P \subseteq\{0,1\}^{*}$ that is a c-set is uniform.

\section{Proposition 2.1}

(1) Let $P \subseteq\{0,1\}^{*}$ be a bar for the binary sequences. Then, $P$ is a c-set if and only if there exists a function $\delta:\{0,1\}^{*} \rightarrow \mathbb{N}$ such that

$(2-1) \quad\left(\forall a \in\{0,1\}^{*}\right)\left[P(a) \leftrightarrow\left(\forall b \in\{0,1\}^{*}\right) \delta(a)=\delta(a * b)\right]$.

(2) $\mathrm{c}-\mathrm{BI} \Longrightarrow \mathrm{c}-\mathrm{FT}$.

Proof $(1)(\Rightarrow)$ Suppose that $P$ is a c-set that is a bar. Let $D \subseteq\{0,1\}^{*}$ be a detachable predicate such that

$$
\left(\forall a \in\{0,1\}^{*}\right)\left[P(a) \leftrightarrow\left(\forall b \in\{0,1\}^{*}\right) D(a * b)\right] .
$$

Define a function $\delta:\{0,1\}^{*} \rightarrow \mathbb{N}$ by

$$
\delta(a) \stackrel{\text { def }}{=} \begin{cases}1 & \text { if } D(a), \\ 0 & \text { otherwise }\end{cases}
$$

Let $a \in\{0,1\}^{*}$, and suppose that $\left(\forall b \in\{0,1\}^{*}\right) \delta(a)=\delta(a * b)$. Since $P$ is a bar, there exists $n \in \mathbb{N}$ such that $P\left(\overline{a * 0^{\omega}} n\right)$, where

$$
0^{\omega} \stackrel{\text { def }}{=} \lambda x .0 \text {. }
$$

Then, obviously $\delta(a)=\delta\left(\overline{a * 0^{\omega}} n\right)=1$. Hence $P(a)$. The converse $P(a) \rightarrow$ $\left(\forall b \in\{0,1\}^{*}\right) \delta(a)=\delta(a * b)$ is obvious.

$(\Leftarrow)$ Let $\delta:\{0,1\}^{*} \rightarrow \mathbb{N}$ be a function that satisfies the condition (2-1). Define a detachable predicate $D \subseteq\{0,1\}^{*}$ by

$$
D(a) \stackrel{\text { def }}{\Longleftrightarrow} \delta(a)=\delta(a *\langle 0\rangle)=\delta(a *\langle 1\rangle) .
$$

Journal of Logic \& Analysis 11:FT3 (2019) 
Obviously we have $P(a) \leftrightarrow\left(\forall b \in\{0,1\}^{*}\right) D(a * b)$.

(2) Assume c-BI. Let $C \subseteq\{0,1\}^{*}$ be a c-set which is a bar of the binary tree. By the first part of this proposition, there exists a function $\delta:\{0,1\}^{*} \rightarrow \mathbb{N}$ such that

$$
C(a) \leftrightarrow\left(\forall b \in\{0,1\}^{*}\right) \delta(a)=\delta(a * b) .
$$

Define a function $\Gamma: \mathbb{N}^{\mathbb{N}} \rightarrow\{0,1\}^{\mathbb{N}}$ by

$$
\Gamma(\alpha) \stackrel{\text { def }}{=} \lambda n \cdot \operatorname{sg}(\alpha(n))
$$

where $\operatorname{sg}(n) \stackrel{\text { def }}{=} \min (1, n)$. Similarly, we define $\Gamma^{*}: \mathbb{N}^{*} \rightarrow\{0,1\}^{*}$. Since $C$ is a bar, we have $\left(\forall \alpha \in \mathbb{N}^{\mathbb{N}}\right)(\exists n \in \mathbb{N}) C(\overline{\Gamma(\alpha)} n)$, ie $\left(\forall \alpha \in \mathbb{N}^{\mathbb{N}}\right)(\exists n \in \mathbb{N}) C\left(\Gamma^{*}(\bar{\alpha} n)\right)$. Define a predicate $P \subseteq \mathbb{N}^{*}$ and a function $\varepsilon: \mathbb{N}^{*} \rightarrow \mathbb{N}$ by:

$$
\begin{aligned}
& P(a) \stackrel{\text { def }}{\Longleftrightarrow} C\left(\Gamma^{*}(a)\right) \\
& \varepsilon(a) \stackrel{\text { def }}{=} \delta\left(\Gamma^{*}(a)\right)
\end{aligned}
$$

Then, $P(a) \leftrightarrow\left(\forall b \in \mathbb{N}^{*}\right) \varepsilon(a)=\varepsilon(a * b)$, so $P$ is a c-bar. Define a predicate $Q \subseteq \mathbb{N}^{*}$ by

$$
Q(a) \stackrel{\text { def }}{\Longleftrightarrow}(\exists N \in \mathbb{N})\left(\forall \alpha \in \mathbb{N}^{\mathbb{N}}\right)(\exists n \leq N) P(a * \bar{\alpha} n) .
$$

Clearly, $P \subseteq Q$. Let $a \in \mathbb{N}^{*}$ and suppose that $(\forall n \in \mathbb{N}) Q(a *\langle n\rangle)$. Then, there exists $N \in \mathbb{N}$ such that for each $i \in\{0,1\}$,

$$
\left(\forall \alpha \in \mathbb{N}^{\mathbb{N}}\right)(\exists n \leq N) P(a *\langle i\rangle * \bar{\alpha} n) .
$$

From the definition of $P$, we see that $Q(a)$. Thus, $Q$ is inductive. Applying c-BI, we obtain $Q(\langle\rangle)$, which implies

$$
(\exists N \in \mathbb{N})\left(\forall \alpha \in\{0,1\}^{\mathbb{N}}\right)(\exists n \leq N) C(\bar{\alpha} n) .
$$

Thus, we can think of c-BI as a generalisation of c-FT to Baire space.

\section{The principle of Brouwer continuity}

We recall the notion of Brouwer-operation from Kreisel and Troelstra [9], which allows us to give a constructive notion of continuity on Baire space which naturally extends the notion of uniform continuity on Cantor space.

Journal of Logic \& Analysis 11:FT3 (2019) 
The predicate $K \subseteq \mathbb{N}^{\mathbb{N}^{*}}$ of Brouwer-operations is inductively defined by the following clauses:

(3-1) $\quad \frac{n \in \mathbb{N}}{\lambda a \cdot n+1 \in K} \quad \frac{\gamma(\langle\rangle)=0 \quad(\forall n \in \mathbb{N}) \lambda a \cdot \gamma(\langle n\rangle * a) \in K}{\gamma \in K}$

Formally, we assume the existence of a predicate $K$ satisfying the axioms K1 - K3; see Introduction 1. If a Brouwer-operation $\gamma \in K$ is introduced by the second clause, we write $\sup _{n \in \mathbb{N}} \gamma_{n}$ for $\gamma$, where

$$
\gamma_{n} \stackrel{\text { def }}{=} \lambda a \cdot \gamma(\langle n\rangle * a)
$$

Let $K_{0}$ be a predicate on $\mathbb{N}^{\mathbb{N}^{*}}$ defined by

$$
\begin{aligned}
K_{0}(\gamma) \stackrel{\text { def }}{\Longleftrightarrow}\left(\forall \alpha \in \mathbb{N}^{\mathbb{N}}\right)(\exists n \in \mathbb{N}) \gamma(\bar{\alpha} n)>0 \wedge \\
\quad\left(\forall a, b \in \mathbb{N}^{*}\right)[\gamma(a)>0 \rightarrow \gamma(a)=\gamma(a * b)] .
\end{aligned}
$$

An element of $K_{0}$ is called a neighbourhood function. Note that every Brouwer-operation is a neighbourhood function.

Lemma 3.1 $K \subseteq K_{0}$.

Proof The proof is by induction on $K$. Details can be found in Troelstra and van Dalen [10, Chapter 4, Proposition 8.5].

The converse of Lemma 3.1 does not necessarily hold; see Lemma 5.2.

By $\mathrm{AC}_{10}$ !, every neighbourhood function $\gamma \in K_{0}$ determines a function $F_{\gamma}: \mathbb{N}^{\mathbb{N}} \rightarrow \mathbb{N}$ by:

$$
F_{\gamma}(\alpha) \stackrel{\text { def }}{=} \gamma\left(\bar{\alpha} \min _{z \in \mathbb{N}}[\gamma(\bar{\alpha} z)>0]\right) \doteq 1
$$

A function $F: \mathbb{N}^{\mathbb{N}} \rightarrow \mathbb{N}$ is $K_{0}$-realisable if there exists a neighbourhood function $\gamma \in K_{0}$ such that $F_{\gamma}=F$. Similarly, a function $F: \mathbb{N}^{\mathbb{N}} \rightarrow \mathbb{N}$ is said to be $K$-realisable if there exists a Brouwer-operation $\gamma \in K$ such that $F_{\gamma}=F$. In both cases, we say that $\gamma$ realises $F$ and write $\gamma \Vdash F$.

We now formulate a continuity principle for Baire space, called the principle of Brouwer continuity (BC): ${ }^{2}$

BC Every pointwise continuous function $F: \mathbb{N}^{\mathbb{N}} \rightarrow \mathbb{N}$ is $K$-realisable.

\footnotetext{
${ }^{2}$ The principle BC is called UCB in [7].
} 
Here, recall that a function $F: \mathbb{N}^{\mathbb{N}} \rightarrow \mathbb{N}$ is pointwise continuous if

$$
\left(\forall \alpha \in \mathbb{N}^{\mathbb{N}}\right)(\exists n \in \mathbb{N})\left(\forall \beta \in \mathbb{N}^{\mathbb{N}}\right) \bar{\beta} n=\bar{\alpha} n \rightarrow F(\beta)=F(\alpha) .
$$

The following argument highlights the difference between pointwise continuity and realisability by neighbourhood functions. Let $K_{1}$ be a predicate on $\mathbb{N}^{\mathbb{N}^{*}}$ defined by

$$
K_{1}(\delta) \stackrel{\text { def }}{\Longleftrightarrow}\left(\forall \alpha \in \mathbb{N}^{\mathbb{N}}\right)(\exists n \in \mathbb{N})\left(\forall a \in \mathbb{N}^{*}\right) \delta(\bar{\alpha} n)=\delta(\bar{\alpha} n * a) .
$$

Note that $K_{0} \subseteq K_{1}$, and the predicate $K_{1}$ represents the class of c-bars.

Every function $\delta \in K_{1}$ determines a pointwise continuous function $F_{\delta}: \mathbb{N}^{\mathbb{N}} \rightarrow \mathbb{N}$ in the following way. For each $\alpha \in \mathbb{N}^{\mathbb{N}}$, define

$$
D_{\alpha} \stackrel{\text { def }}{=}\{m \in \mathbb{N} \mid \delta(\bar{\alpha} m) \neq \delta(\bar{\alpha}(m+1))\} \cup\{1\} .
$$

Then, $D_{\alpha}$ is bounded because $\delta$ determines a c-bar. By $\mathrm{AC}_{10}$ !, defined a function $F_{\delta}: \mathbb{N}^{\mathbb{N}} \rightarrow \mathbb{N}$ by

$$
F_{\delta}(\alpha) \stackrel{\text { def }}{=} \delta\left(\bar{\alpha}\left(\max D_{\alpha}+1\right)\right) .
$$

To see that $F_{\delta}$ is pointwise continuous, let $\alpha \in \mathbb{N}^{\mathbb{N}}$. Then, there exists $n \in \mathbb{N}$ such that $\left(\forall a \in \mathbb{N}^{*}\right) \delta(\bar{\alpha} n)=\delta(\bar{\alpha} n * a)$. Then, for any $\beta \in \bar{\alpha} n$, we have $D_{\beta}=D_{\alpha}$, and so $F_{\delta}(\alpha)=F_{\delta}(\beta)$. Hence $F_{\delta}$ is pointwise continuous. Conversely, every pointwise continuous function $F: \mathbb{N}^{\mathbb{N}} \rightarrow \mathbb{N}$ arises in this way from a function $\delta \in K_{1}$ by setting

$$
\delta(a) \stackrel{\text { def }}{=} F\left(a * 0^{\omega}\right) .
$$

In the rest of this section, we relate $\mathrm{BC}$ to the uniform continuity principle $\mathrm{UC}$.

First, we adjust the notion of Brouwer-operation to the functions on Cantor space. The predicate $K_{C} \subseteq \mathbb{N}^{\{0,1\}^{*}}$ of Brouwer-operations on Cantor space is inductively defined by the following clauses:

$$
\frac{n \in \mathbb{N}}{\lambda a . n+1 \in K_{C}} \quad \frac{\gamma(\langle\rangle)=0 \quad(\forall i \in\{0,1\}) \lambda a \cdot \gamma(\langle i\rangle * a) \in K_{C}}{\gamma \in K_{C}}
$$

Each Brouwer-operation $\gamma \in K_{C}$ determines a continuous function $F_{\gamma}:\{0,1\}^{\mathbb{N}} \rightarrow \mathbb{N}$ as in equation (3-2). The notion of $K_{C}$-realisable function from $\{0,1\}^{\mathbb{N}}$ to $\mathbb{N}$ is similarly defined.

In the following proposition, recall that a function $F:\{0,1\}^{\mathbb{N}} \rightarrow \mathbb{N}$ is uniformly continuous if

$$
(\exists n \in \mathbb{N})\left(\forall \alpha, \beta \in\{0,1\}^{\mathbb{N}}\right) \bar{\alpha} n=\bar{\beta} n \rightarrow F(\alpha)=F(\beta) .
$$


Proposition 3.2 A function $F:\{0,1\}^{\mathbb{N}} \rightarrow \mathbb{N}$ is uniformly continuous if and only if $F$ is $K_{C}$-realisable.

Proof $(\Rightarrow)$ Define a predicate $A \subseteq \mathbb{N}$ by

$$
\begin{array}{r}
A(n) \stackrel{\text { def }}{\Longleftrightarrow}\left(\forall F \in \mathbb{N}^{\left(\{0,1\}^{\mathbb{N}}\right)}\right)\left[\left(\forall \alpha, \beta \in\{0,1\}^{\mathbb{N}}\right)[\bar{\alpha} n=\bar{\beta} n \rightarrow F(\alpha)=F(\beta)]\right. \\
\left.\rightarrow\left(\exists \gamma \in K_{C}\right) \gamma \Vdash F\right] .
\end{array}
$$

It suffices to show that $A(n)$ for all $n \in \mathbb{N}$, which is proved by induction.

$n=0$ : Then $\lambda a \cdot F\left(0^{\omega}\right)+1$ realises $F$.

$n=k+1:$ Let $F:\{0,1\}^{\mathbb{N}} \rightarrow \mathbb{N}$ be a function such that

$$
\left(\forall \alpha, \beta \in\{0,1\}^{\mathbb{N}}\right) \bar{\alpha} n=\bar{\beta} n \rightarrow F(\alpha)=F(\beta) .
$$

By induction hypothesis, there exist $\gamma_{0}, \gamma_{1} \in K_{C}$ such that for each $i \in\{0,1\}$ the Brouwer-operation $\gamma_{i}$ realises a function $F_{i}:\{0,1\}^{\mathbb{N}} \rightarrow \mathbb{N}$ given by

$$
F_{i}(\alpha) \stackrel{\text { def }}{=} F(\langle i\rangle * \alpha) \text {. }
$$

Define $\gamma \in K_{C}$ by $\gamma(\langle\rangle) \stackrel{\text { def }}{=} 0$, and $\lambda a . \gamma(\langle i\rangle * a) \stackrel{\text { def }}{=} \gamma_{i}$ for $i \in\{0,1\}$. Let $\alpha \in\{0,1\}^{\mathbb{N}}$, and put $i=\alpha(0)$. Since $\gamma_{i} \Vdash F_{i}$, there exists $k \in \mathbb{N}$ such that $\gamma_{i}\left(\overline{\alpha_{\geq 1}} k\right)=F_{i}\left(\alpha_{\geq 1}\right)+1$, where $\alpha_{\geq 1} \stackrel{\text { def }}{=} \lambda n . \alpha(n+1)$. Then $\gamma(\bar{\alpha}(k+1))=F(\alpha)+1$. Therefore $\gamma$ realises $F$.

$(\Leftarrow)$ Suppose that $F$ is realised by $\gamma \in K_{C}$. We show by induction on $K_{C}$ that

$$
\left(\forall F \in \mathbb{N}^{\{0,1\}^{\mathbb{N}}}\right) \gamma \Vdash F \rightarrow \text { " } F \text { is uniformly continuous" }
$$

where " $F$ is uniformly continuous" is the formula of the form (3-4).

$\gamma=\lambda a . n+1:$ Then $\gamma$ realises the constant function $\lambda \alpha . n$, which is uniformly continuous.

$\gamma(\langle\rangle)=0 \wedge(\forall i \in\{0,1\}) \lambda a \cdot \gamma(\langle i\rangle * a) \in K_{C}$ : Let $F:\{0,1\}^{\mathbb{N}} \rightarrow \mathbb{N}$ be a function such that $\gamma \Vdash F$. Then, for each $i \in\{0,1\}$ we have $\lambda a . \gamma(\langle i\rangle * a) \Vdash F_{i}$, where $F_{i}$ is defined as in (3-5). By induction hypothesis, $F_{i}$ is uniformly continuous for each $i \in\{0,1\}$. Hence $F$ is uniformly continuous. 


\section{Equivalence of $\mathrm{c}-\mathrm{BI}$ and $\mathrm{BC}$}

The aim of this section is to prove the following equivalence.

Theorem $4.1 \quad \mathrm{c}-\mathrm{BI} \Longleftrightarrow \mathrm{BC}$.

First, we prove the direction $(\Rightarrow)$.

Proposition $4.2 \mathrm{c}-\mathrm{BI} \Longrightarrow \mathrm{BC}$.

Proof Assume c-BI. Let $F: \mathbb{N}^{\mathbb{N}} \rightarrow \mathbb{N}$ be a pointwise continuous function. Define a function $\delta: \mathbb{N}^{\mathbb{N}} \rightarrow \mathbb{N}$ and a predicate $P \subseteq \mathbb{N}^{*}$ by:

$$
\begin{aligned}
& \delta(a) \stackrel{\text { def }}{=} F\left(a * 0^{\omega}\right) \\
& P(a) \stackrel{\text { def }}{\Longleftrightarrow}\left(\forall b \in \mathbb{N}^{*}\right) \delta(a)=\delta(a * b)
\end{aligned}
$$

Since $F$ is pointwise continuous, $P$ is a c-bar. Define a predicate $Q \subseteq \mathbb{N}^{*}$ by

(4-1) $Q(a) \stackrel{\text { def }}{\Longleftrightarrow}(\exists \gamma \in K)\left(\forall b \in \mathbb{N}^{*}\right) \gamma(b)>0 \rightarrow P(a * b) \wedge \gamma(b)=\delta(a * b)+1$.

We show that

(1) $P \subseteq Q$, and

(2) $Q$ is inductive.

(1) Let $a \in \mathbb{N}^{*}$ such that $P(a)$. Define $\gamma \in K$ by $\gamma \stackrel{\text { def }}{=} \lambda b . \delta(a)+1$. Then, $\gamma$ is a witness of the existential quantifier in (4-1). Thus $Q(a)$.

(2) Let $a \in \mathbb{N}^{*}$ and suppose that $(\forall n \in \mathbb{N}) Q(a *\langle n\rangle)$. By $\mathrm{AC}_{01}$, there exists a sequence $\left(\gamma_{n}\right)_{n \in \mathbb{N}}$ of Brouwer-operations such that

$$
(\forall n \in \mathbb{N})\left(\forall b \in \mathbb{N}^{*}\right) \gamma_{n}(b)>0 \rightarrow P(a *\langle n\rangle * b) \wedge \gamma_{n}(b)=\delta(a *\langle n\rangle * b)+1 .
$$

Put $\gamma \stackrel{\text { def }}{=} \sup _{n \in \mathbb{N}} \gamma_{n}$. Let $b \in \mathbb{N}^{*}$, and suppose that $\gamma(b)>0$. Then, there exist $n \in \mathbb{N}$ and $b^{\prime} \in \mathbb{N}^{*}$ such that $b=\langle n\rangle * b^{\prime} \wedge \gamma_{n}\left(b^{\prime}\right)>0$. Thus, $P\left(a *\langle n\rangle * b^{\prime}\right) \wedge \gamma_{n}\left(b^{\prime}\right)=$ $\delta\left(a *\langle n\rangle * b^{\prime}\right)+1$, that is $P(a * b) \wedge \gamma(b)=\delta(a * b)+1$. Hence $Q(a)$.

By c-BI, we obtain $Q(\langle\rangle)$, ie there exists $\gamma \in K$ such that

$$
\left(\forall a \in \mathbb{N}^{*}\right) \gamma(a)>0 \rightarrow P(a) \wedge \gamma(a)=\delta(a)+1 .
$$

Therefore $\gamma$ realises $F$.

To prove the direction $(\Leftarrow)$ of Theorem 4.1 , we need some preliminaries. 
Lemma 4.3 (Kreisel and Troelstra [9, Theorem 3.1.2]) Let $Q$ be a predicate on $\mathbb{N}^{*}$. Then

$$
(\forall \gamma \in K)\left[P_{\gamma} \subseteq Q \wedge\left(\forall a \in \mathbb{N}^{*}\right)[(\forall n \in \mathbb{N}) Q(a *\langle n\rangle) \rightarrow Q(a)] \rightarrow Q(\langle\rangle)\right]
$$

where $P_{\gamma} \stackrel{\text { def }}{=}\left\{a \in \mathbb{N}^{*} \mid \gamma(a)>0\right\}$.

Proof See Kreisel and Troelstra [9, Theorem 3.1.2].

We prove the following two lemmas for the sake of completeness.

Lemma 4.4 (Troelstra and van Dalen [10, Exercise 4.8.5])

$$
(\forall \gamma \in K)\left(\forall \gamma^{\prime} \in K_{0}\right)\left[\left(\forall a \in \mathbb{N}^{*}\right)\left[\gamma(a)>0 \rightarrow \gamma^{\prime}(a)>0\right] \rightarrow \gamma^{\prime} \in K\right] .
$$

Proof By induction on $K$.

$\gamma=\lambda a . n+1:$ For any $\gamma^{\prime} \in K_{0}$, if $\left(\forall a \in \mathbb{N}^{*}\right) \gamma(a)>0 \rightarrow \gamma^{\prime}(a)>0$, then $\gamma^{\prime}$ is a constant function with a positive value. Thus $\gamma^{\prime} \in K$.

$\gamma=\sup _{n \in \mathbb{N}} \gamma_{n}$ : Let $\gamma^{\prime} \in K_{0}$ and suppose that $\left(\forall a \in \mathbb{N}^{*}\right) \gamma(a)>0 \rightarrow \gamma^{\prime}(a)>0$. Then, for each $n \in \mathbb{N}$, we have $\gamma_{n}^{\prime} \stackrel{\text { def }}{=} \lambda a \cdot \gamma^{\prime}(\langle n\rangle * a) \in K_{0}$ and $\left(\forall a \in \mathbb{N}^{*}\right) \gamma_{n}(a)>$ $0 \rightarrow \gamma_{n}^{\prime}(a)>0$. By induction hypothesis, we have $\gamma_{n}^{\prime} \in K$ for all $n \in \mathbb{N}$. Since $\gamma^{\prime}=\sup _{n \in \mathbb{N}} \gamma_{n}^{\prime}$, we conclude $\gamma^{\prime} \in K$.

Lemma 4.5 (Troelstra and van Dalen [10, Exercises 4.8.6])

$$
(\forall \gamma \in K) \lambda a \cdot \gamma(a) \cdot \operatorname{sg}(|a| \doteq \gamma(a)) \in K .
$$

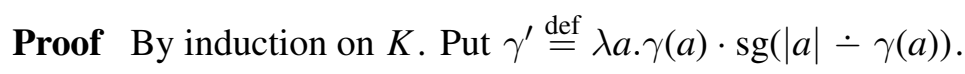

$\gamma=\lambda a . n+1$ : This follows from Kreisel and Troelstra [9, Theorem 3.2.2 (iv), (vi)]. Alternatively, it is clear that $\lambda a \cdot(n+1) \cdot \operatorname{sg}(|a| \doteq(n+1))$ is introduced in $K$ by $(n+2)$-times applications of the second clause of (3-1).

$\gamma=\sup _{n \in \mathbb{N}} \gamma_{n}$ : By induction hypothesis, we have $\gamma_{n}^{\prime} \in K$ for all $n \in \mathbb{N}$. Put $\xi \stackrel{\text { def }}{=} \sup _{n \in \mathbb{N}} \gamma_{n}^{\prime} \in K$. Let $a \in \mathbb{N}^{*}$ and suppose that $\xi(a)>0$. Then, there exist $n \in \mathbb{N}$ and $a^{\prime} \in \mathbb{N}^{*}$ such that $a=\langle n\rangle * a^{\prime}$ and $\gamma_{n}^{\prime}\left(a^{\prime}\right)>0$. Thus $\gamma^{\prime}(a)=\gamma(a) \cdot \operatorname{sg}(|a| \doteq \gamma(a))>0$. Clearly, we have $\gamma^{\prime} \in K_{0}$. Hence $\gamma^{\prime} \in K$ by Lemma 4.4.

We now prove the direction $(\Leftarrow)$ of Theorem 4.1 . 
Proposition 4.6 $\mathrm{BC} \Longrightarrow \mathrm{c}-\mathrm{BI}$.

Proof Let $P \subseteq \mathbb{N}^{*}$ be a bar, and let $\delta: \mathbb{N}^{*} \rightarrow \mathbb{N}$ be a function such that $P(a) \leftrightarrow$ $\left(\forall b \in \mathbb{N}^{*}\right) \delta(a)=\delta(a * b)$. Let $Q \subseteq \mathbb{N}^{*}$ be an inductive predicate such that $P \subseteq Q$. Define a function $F: \mathbb{N}^{\mathbb{N}} \rightarrow \mathbb{N}$ by

$$
F(\alpha) \stackrel{\text { def }}{=} \max D_{\alpha}
$$

where $D_{\alpha}$ is given by (3-3). Then, $F$ is pointwise continuous. By BC, there exists a Brouwer-operation $\gamma \in K$ such that $F_{\gamma}=F$. By Lemma 4.5, we may assume that $\left(\forall a \in \mathbb{N}^{*}\right) \gamma(a)>0 \rightarrow|a|>\gamma(a)$. Let $a \in \mathbb{N}^{*}$ such that $\gamma(a)>0$. Let $b \in \mathbb{N}^{*}$. Then, $|a|>\gamma(a) \wedge \gamma(a)=\gamma(a * b)$ so that

$$
|a|>\max D_{a * 0^{\omega}}+1=\max D_{a * b * 0^{\omega}}+1 .
$$

Thus $\delta(a)=\delta(a * b)$. Hence $P(a)$, and so $Q(a)$. By Proposition 4.3, we obtain $Q(\langle\rangle)$.

This completes the proof of Theorem 4.1. We note that the structure of the proof of Proposition 4.6 is quite similar to the proof of the implication UC $\Longrightarrow$ c-FT by Berger [3, Proposition 2].

\section{Characterisation of bar inductions by continuity principles}

We show that the decidable bar induction and the monotone bar induction can be characterised by statements similar to $\mathrm{BC}$.

\subsection{Decidable bar induction}

The decidable bar induction $\mathrm{BI}_{\mathbf{D}}$ is the following statement:

BI For any detachable bar $P \subseteq \mathbb{N}^{*}$ and a predicate $Q \subseteq \mathbb{N}^{*}$, if $P \subseteq Q$ and $Q$ is inductive, then $Q(\langle\rangle)$.

We relate $\mathrm{BI}_{\mathbf{D}}$ to two notions of continuity.

First, recall that in Section 3 we defined a function $F: \mathbb{N}^{\mathbb{N}} \rightarrow \mathbb{N}$ to be $K_{0}$-realisable if there exists a neighbourhood function $\gamma \in K_{0}$ such that $F_{\gamma}=F$.

Next, given a function $F: \mathbb{N}^{\mathbb{N}} \rightarrow \mathbb{N}$, a function $g: \mathbb{N}^{\mathbb{N}} \rightarrow \mathbb{N}$ is a modulus of $F$ if

$$
\left(\forall \alpha \in \mathbb{N}^{\mathbb{N}}\right)(\forall \beta \in \bar{\alpha} g(\alpha)) F(\beta)=F(\alpha) .
$$


The following lemma is due to Beeson [1, Chapter VI, Section 8, Exercise 8]. ${ }^{3}$

Lemma 5.1 A function $F: \mathbb{N}^{\mathbb{N}} \rightarrow \mathbb{N}$ is $K_{0}$-realisable if and only if $F$ has a pointwise continuous modulus of continuity.

Proof Suppose that $F$ is realised by $\gamma \in K_{0}$. By $\mathrm{AC}_{10}$ !, define $g: \mathbb{N}^{\mathbb{N}} \rightarrow \mathbb{N}$ by

$$
g(\alpha) \stackrel{\text { def }}{=} \min \{n \in \mathbb{N} \mid \gamma(\bar{\alpha} n)>0\} .
$$

Then, $g$ is a modulus of $F$. It is also clear that $g$ is pointwise continuous.

Conversely, suppose that $F$ has a pointwise continuous modulus $g: \mathbb{N}^{\mathbb{N}} \rightarrow \mathbb{N}$. Define a function $\gamma: \mathbb{N}^{*} \rightarrow \mathbb{N}$ by

$$
\gamma(a) \stackrel{\text { def }}{=} \begin{cases}F\left(a * 0^{\omega}\right)+1 & \text { if }\left(\exists a^{\prime} \preccurlyeq a\right)\left|a^{\prime}\right| \geq g\left(a^{\prime} * 0^{\omega}\right), \\ 0 & \text { otherwise. }\end{cases}
$$

We show that $\gamma \in K_{0}$ and that $\gamma$ realises $F$. Let $\alpha \in \mathbb{N}^{\mathbb{N}}$. Since $g$ is pointwise continuous, there exists $n \in \mathbb{N}$ such that $n \geq g\left(\bar{\alpha} n * 0^{\omega}\right)$. Since $g$ is a modulus of $F$,

$$
\gamma(\bar{\alpha} n)=F\left(\bar{\alpha} n * 0^{\omega}\right)+1=F(\alpha)+1 .
$$

Next, let $a \in \mathbb{N}^{*}$ and suppose that $\gamma(a)>0$. Then, there exists $a^{\prime} \preccurlyeq a$ such that $\left|a^{\prime}\right| \geq g\left(a^{\prime} * 0^{\omega}\right)$. Thus, $F\left(a^{\prime} * 0^{\omega}\right)=F\left(a * 0^{\omega}\right)=F\left(a * b * 0^{\omega}\right)$ for all $b \in \mathbb{N}^{*}$. Hence, $\left(\forall b \in \mathbb{N}^{*}\right) \gamma(a)=\gamma(a * b)$. Therefore, $\gamma \in K_{0}$ and $\gamma$ realises $F$.

We recall the following result from Troelstra and van Dalen [10, Proposition 8.13 (i)].

Lemma 5.2 $\mathrm{BI}_{\mathbf{D}} \Longleftrightarrow K=K_{0}$.

Proof See Troelstra and van Dalen [10, Proposition 8.13 (i)].

Proposition 5.3 The following are equivalent.

(1) $\mathrm{BI}_{\mathbf{D}}$.

(2) Every $K_{0}$-realisable function $F: \mathbb{N}^{\mathbb{N}} \rightarrow \mathbb{N}$ is $K$-realisable.

(3) Every function $F: \mathbb{N}^{\mathbb{N}} \rightarrow \mathbb{N}$ which has a pointwise continuous modulus is $K$ realisable.

\footnotetext{
${ }^{3}$ In Beeson [1], neighbourhood functions are called associates.
} 
Proof In view of Lemma 5.1 and Lemma 5.2, it suffices to show that (2) implies $K_{0} \subseteq K$.

Assume (2). Let $\gamma \in K_{0}$. Define a neighbourhood function $\gamma^{\prime} \in K_{0}$ by

$$
\gamma^{\prime}(a) \stackrel{\text { def }}{=} \begin{cases}0 & \text { if }(\forall b \preccurlyeq a) \gamma(b)=0, \\ \min \{|b| \mid b \preccurlyeq a \wedge \gamma(b)>0\}+1 & \text { otherwise. }\end{cases}
$$

By the assumption, there exists a Brouwer-operation $\xi \in K$ that realises the function $F_{\gamma^{\prime}}: \mathbb{N}^{\mathbb{N}} \rightarrow \mathbb{N}$ induced by $\gamma^{\prime}$. By Lemma 4.5 , we may assume that

$$
\left(\forall a \in \mathbb{N}^{*}\right) \xi(a)>0 \rightarrow|a|>\xi(a) .
$$

Let $a \in \mathbb{N}^{*}$, and suppose that $\xi(a)>0$. Then, $|a|>\xi(a)=F_{\gamma^{\prime}}\left(a * 0^{\omega}\right)+1$. Thus, there exists $k \in \mathbb{N}$ such that $\gamma^{\prime}\left(\overline{a * 0^{\omega}} k\right)=F_{\gamma^{\prime}}\left(a * 0^{\omega}\right)+1$. By the definition of $\gamma^{\prime}$, there exists $b \preccurlyeq \overline{a * 0^{\omega}} k$ such that $\gamma(b)>0$ and $|b|+1=\gamma^{\prime}\left(\overline{a * 0^{\omega}} k\right)$. Hence $b \preccurlyeq a$ so that $\gamma(a)>0$. By Lemma 4.4, we obtain $\gamma \in K$.

Remark 5.4 The decidable fan theorem is a version of the fan theorem formulated with respect to decidable bars on $\{0,1\}^{*}$. Berger [2] showed that the decidable fan theorem and the following statement are equivalent:

Every function $F:\{0,1\}^{\mathbb{N}} \rightarrow \mathbb{N}$ which has a pointwise continuous modulus is uniformly continuous.

Here, a modulus of $F:\{0,1\}^{\mathbb{N}} \rightarrow \mathbb{N}$ is similarly defined as in (5-1). Proposition 5.3 says that this characterisation naturally extends to the decidable bar induction.

\subsection{Monotone bar induction}

The monotone bar induction $\mathrm{BI}_{\mathbf{M}}$ is the following statement:

BI $_{\mathbf{M}}$ For any monotone bar $P \subseteq \mathbb{N}^{*}$ and a predicate $Q \subseteq \mathbb{N}^{*}$, if $P \subseteq Q$ and $Q$ is inductive, then $Q(\langle\rangle)$.

Here, a bar $P \subseteq \mathbb{N}^{*}$ is monotone if $\left(\forall a, b \in \mathbb{N}^{*}\right) P(a) \rightarrow P(a * b)$.

A predicate $R \subseteq \mathbb{N}^{\mathbb{N}} \times \mathbb{N}$ is said to be locally continuous if

$$
\left(\forall \alpha \in \mathbb{N}^{\mathbb{N}}\right)(\exists x \in \mathbb{N})(\exists y \in \mathbb{N})(\forall \beta \in \bar{\alpha} x) R(\beta, y) .
$$

Given a locally continuous predicate $R \subseteq \mathbb{N}^{\mathbb{N}} \times \mathbb{N}$, we say that a function $F: \mathbb{N}^{\mathbb{N}} \rightarrow \mathbb{N}$ refines $R$ if $\left(\forall \alpha \in \mathbb{N}^{\mathbb{N}}\right) R(\alpha, F(\alpha))$, ie $F$ is a choice function of $R$. 
Proposition 5.5 The following are equivalent.

(1) $\mathrm{BI}_{\mathbf{M}}$.

(2) Every locally continuous predicate $R \subseteq \mathbb{N}^{\mathbb{N}} \times \mathbb{N}$ has a $K$-realisable function which refines $R$.

Proof (1) $\Rightarrow$ (2) Assume $\mathrm{BI}_{\mathbf{M}}$. Let $R \subseteq \mathbb{N}^{\mathbb{N}} \times \mathbb{N}$ be a locally continuous predicate. Define a predicate $P \subseteq \mathbb{N}^{*}$ by

$$
P(a) \stackrel{\text { def }}{\Longleftrightarrow}(\exists x \in \mathbb{N})\left(\forall \alpha \in \mathbb{N}^{\mathbb{N}}\right) \alpha \in a \rightarrow R(\alpha, x) .
$$

Clearly, $P$ is a monotone bar. Define a predicate $Q \subseteq \mathbb{N}^{*}$ by

$$
\begin{aligned}
Q(a) \stackrel{\text { def }}{\Longleftrightarrow}(\exists \gamma \in K)\left(\forall \alpha \in \mathbb{N}^{\mathbb{N}}\right)\left(\forall b \in \mathbb{N}^{*}\right) & \\
{[\gamma(b)} & >0 \wedge \alpha \in a * b] \rightarrow R(\alpha, \gamma(b)-1) .
\end{aligned}
$$

We show that

(1) $P \subseteq Q$, and

(2) $Q$ is inductive.

(1) Let $a \in \mathbb{N}^{*}$ such that $P(a)$. Then, there exists $n \in \mathbb{N}$ such that $\left(\forall \alpha \in \mathbb{N}^{\mathbb{N}}\right) \alpha \in$ $a \rightarrow R(\alpha, n)$. Put $\gamma \stackrel{\text { def }}{=} \lambda a . n+1$, which is in $K$. Then, $\gamma$ is a witness of the existential quantifier in (5-2). Thus $Q(a)$.

(2) Let $a \in \mathbb{N}^{*}$ and suppose that $(\forall n \in \mathbb{N}) Q(a *\langle n\rangle)$. By $\mathrm{AC}_{01}$, there exists a sequence $\left(\gamma_{n}\right)_{n \in \mathbb{N}}$ of Brouwer-operations such that

$$
(\forall n \in \mathbb{N})\left(\forall \alpha \in \mathbb{N}^{\mathbb{N}}\right)\left(\forall b \in \mathbb{N}^{*}\right)\left[\gamma_{n}(b)>0 \wedge \alpha \in a *\langle n\rangle * b \rightarrow R\left(\alpha, \gamma_{n}(b) \doteq 1\right)\right]
$$

Put $\gamma \stackrel{\text { def }}{=} \sup _{n \in \mathbb{N}} \gamma_{n}$. Let $\alpha \in \mathbb{N}^{\mathbb{N}}$ and $b \in \mathbb{N}^{*}$, and suppose that $\gamma(b)>0$ and $\alpha \in a * b$. Then, there exist $n \in \mathbb{N}$ and $b^{\prime} \in \mathbb{N}^{*}$ such that $b=\langle n\rangle * b^{\prime} \wedge \gamma_{n}\left(b^{\prime}\right)>0$. Thus, $\alpha \in a *\langle n\rangle * b^{\prime}$, so $R\left(\alpha, \gamma_{n}\left(b^{\prime}\right) \doteq 1\right)$, that is $R(\alpha, \gamma(b) \doteq 1)$. Hence $Q(a)$.

By $\mathrm{BI}_{\mathbf{M}}$, we obtain $Q(\langle\rangle)$, ie there exists a Brouwer-operation $\gamma \in K$ such that

$$
\left(\forall \alpha \in \mathbb{N}^{\mathbb{N}}\right)\left(\forall a \in \mathbb{N}^{*}\right) \gamma(a)>0 \wedge \alpha \in a \rightarrow R(\alpha, \gamma(a) \dot{-1}) .
$$

Thus, the function $F_{\gamma}: \mathbb{N}^{\mathbb{N}} \rightarrow \mathbb{N}$ induced by $\gamma$ refines $R$.

(2) $\Rightarrow$ (1) Assume (2). Let $P$ be a monotone bar, and let $Q \subseteq \mathbb{N}^{*}$ be an inductive predicate such that $P \subseteq Q$. Define a predicate $R \subseteq \mathbb{N}^{\mathbb{N}} \times \mathbb{N}$ by

$$
R(\alpha, x) \stackrel{\text { def }}{\Longleftrightarrow} P(\bar{\alpha} x) .
$$


Then $R$ is clearly locally continuous. Thus, there exists a Brouwer-operation $\gamma \in K$ such that

$$
\left(\forall \alpha \in \mathbb{N}^{\mathbb{N}}\right) P\left(\bar{\alpha} F_{\gamma}(\alpha)\right) .
$$

By Lemma 4.5, we may assume that $\left(\forall a \in \mathbb{N}^{*}\right) \gamma(a)>0 \rightarrow|a|>\gamma(a)$. Let $a \in \mathbb{N}^{*}$ such that $\gamma(a)>0$. Then, we have $\overline{a * 0^{\omega}} \gamma(a) \preccurlyeq \overline{a * 0^{\omega}}|a|=a$. Since $P\left(\overline{a * 0^{\omega}}(\gamma(a)-1)\right)$ and $P$ is monotone, we have $P(a)$, and thus $Q(a)$. Since $Q$ is inductive, we obtain $Q(\langle\rangle)$ by Proposition 4.3.

\section{Continuity axioms}

A continuity axiom states that if we have $\left(\forall \alpha \in \mathbb{N}^{\mathbb{N}}\right)(\exists x \in \mathbb{N}) R(\alpha, x)$, then the dependence of $x \in \mathbb{N}$ on $\alpha \in \mathbb{N}^{\mathbb{N}}$ is continuous. By varying the strength of continuity with which $x$ depends on $\alpha$, we obtain several principles. The following continuity axioms are well known; see Troelstra and van Dalen [10, Chapter 4, Section 6 and Section 8].

BC-N $\left(\forall \alpha \in \mathbb{N}^{\mathbb{N}}\right)(\exists x \in \mathbb{N}) R(\alpha, x) \rightarrow(\exists \gamma \in K)\left(\forall \alpha \in \mathbb{N}^{\mathbb{N}}\right) R\left(\alpha, F_{\gamma}(\alpha)\right)$.

C-N $\quad\left(\forall \alpha \in \mathbb{N}^{\mathbb{N}}\right)(\exists x \in \mathbb{N}) R(\alpha, x) \rightarrow\left(\exists \gamma \in K_{0}\right)\left(\forall \alpha \in \mathbb{N}^{\mathbb{N}}\right) R\left(\alpha, F_{\gamma}(\alpha)\right)$.

WC-N $\left(\forall \alpha \in \mathbb{N}^{\mathbb{N}}\right)(\exists x \in \mathbb{N}) R(\alpha, x) \rightarrow\left(\forall \alpha \in \mathbb{N}^{\mathbb{N}}\right)(\exists x, y \in \mathbb{N})(\forall \beta \in \bar{\alpha} x) R(\beta, y)$.

Here, $F_{\gamma}$ is the function $F_{\gamma}: \mathbb{N}^{\mathbb{N}} \rightarrow \mathbb{N}$ induced by $\gamma \in K$ (or $\gamma \in K_{0}$ ). The notions of continuity that correspond to BC-N, C-N, and WC-N are that of $K$-realisability, $K_{0}$-realisability, and local continuity, respectively.

The following is immediate from Proposition 5.3 and Proposition 5.5.

\section{Theorem 6.1}

(1) $\mathrm{BC}-\mathrm{N} \Longleftrightarrow \mathrm{BI}_{\mathbf{D}}+\mathrm{C}-\mathrm{N}$.

(2) $\mathrm{BC}-\mathrm{N} \Longleftrightarrow \mathrm{BI}_{\mathbf{M}}+\mathrm{WC}-\mathrm{N}$.

Remark 6.2 Theorem 6.1 is not new. The equivalence (1) can be found in Troelstra and van Dalen [10, Chapter 4, Proposition 8.13 (iii)], and the equivalence (2) was shown by Kreisel and Troelstra [9, Theorem 5.6.3 (ii)]. However, Proposition 5.3 and Proposition 5.5 make these equivalences obvious. Moreover, they clarify the complementary roles of various versions of bar induction and continuity axiom, which is one of the main contributions of the present work.

We can formulate a continuity axiom with respect to the notion of pointwise continuity. The principle of pointwise continuity (PC-N) is the following statement: 
PC-N $\left(\forall \alpha \in \mathbb{N}^{\mathbb{N}}\right)(\exists x \in \mathbb{N}) R(\alpha, x)$

$$
\rightarrow\left(\exists \delta \in \mathbb{N}^{\mathbb{N}^{*}}\right)\left(\forall \alpha \in \mathbb{N}^{\mathbb{N}}\right)(\exists x \in \mathbb{N})\left(\forall a \in \mathbb{N}^{*}\right) \delta(\bar{\alpha} x)=\delta(\bar{\alpha} x * a) \wedge R(\alpha, \delta(\bar{\alpha} x))
$$

The principle PC-N asserts the existence of a pointwise continuous choice function from the assumption $\left(\forall \alpha \in \mathbb{N}^{\mathbb{N}}\right)(\exists x \in \mathbb{N}) R(\alpha, x)$. One can show that PC-N is equivalent to the following statement:

$$
\begin{aligned}
&\left(\forall \alpha \in \mathbb{N}^{\mathbb{N}}\right)(\exists x \in \mathbb{N}) R(\alpha, x) \\
& \rightarrow\left(\exists \delta \in \mathbb{N}^{\mathbb{N}^{*}}\right)\left(\forall \alpha \in \mathbb{N}^{\mathbb{N}}\right)(\exists x \in \mathbb{N})\left(\forall a \in \mathbb{N}^{*}\right)(\delta(\bar{\alpha} x)=\delta(\bar{\alpha} x * a) \\
&\wedge(\forall \beta \in \bar{\alpha} x) R(\beta, \delta(\bar{\alpha} x)))
\end{aligned}
$$

The following equivalence is immediate from Theorem 4.1.

Proposition 6.3 $\mathrm{BC}-\mathrm{N} \Longleftrightarrow \mathrm{c}-\mathrm{BI}+\mathrm{PC}-\mathrm{N}$.

\section{$7 \quad \Pi_{1}^{0}$ bar induction}

The $\Pi_{1}^{0}$ bar induction ( $\Pi_{1}^{0}$-BI) is defined with respect to a bar that is a $\Pi_{1}^{0}$-set, where a predicate $P \subseteq \mathbb{N}^{*}$ is a $\Pi_{1}^{0}$-set if there is a detachable predicate $D \subseteq \mathbb{N}^{*} \times \mathbb{N}$ such that

$$
\left(\forall a \in \mathbb{N}^{*}\right)[P(a) \leftrightarrow(\forall n \in \mathbb{N}) D(a, n)] .
$$

Specifically, $\Pi_{1}^{0}-\mathrm{BI}$ is the following statement:

$\Pi_{1}^{0}$-BI For any $\Pi_{1}^{0}$-bar $P \subseteq \mathbb{N}^{*}$ and a predicate $Q \subseteq \mathbb{N}^{*}$, if $P \subseteq Q$ and $Q$ is inductive, then $Q(\langle\rangle)$.

Note that every c-bar is a $\Pi_{1}^{0}$-set modulo the coding of finite sequences in $\mathbb{N}$. Thus, $\Pi_{1}^{0}$-BI implies c-BI. We show, however, that $\Pi_{1}^{0}$-BI is not an intuitionistic principle.

Recall that LLPO (the lesser limited principle of omniscience) is $\Sigma_{1}^{0}$ De Morgan's Law, ie for any $\alpha, \beta \in \mathbb{N}^{\mathbb{N}}$,

$$
\begin{aligned}
\neg[(\exists n \in \mathbb{N}) \alpha(n) \neq 0 \wedge(\exists n \in \mathbb{N}) \beta(n) & \neq 0] \rightarrow \\
& \neg(\exists n \in \mathbb{N}) \alpha(n) \neq 0 \vee \neg(\exists n \in \mathbb{N}) \beta(n) \neq 0 .
\end{aligned}
$$

Proposition 7.1 $\Pi_{1}^{0}$-BI implies LLPO.

Journal of Logic \& Analysis 11:FT3 (2019) 
Proof Assume $\Pi_{1}^{0}$-BI. Let $\alpha, \beta \in \mathbb{N}^{\mathbb{N}}$, and suppose that

$$
\neg[(\exists n \in \mathbb{N}) \alpha(n) \neq 0 \wedge(\exists n \in \mathbb{N}) \beta(n) \neq 0] .
$$

Define a predicate $P \subseteq \mathbb{N}^{*}$ by

$$
P \stackrel{\text { def }}{=}\{\langle n\rangle \mid \alpha(n)=0\} \cup\{\langle\rangle \mid(\forall n \in \mathbb{N}) \beta(n)=0\} .
$$

Note that $P$ is a $\Pi_{1}^{0}$-set. We show that $P$ is a bar. Let $\gamma \in \mathbb{N}^{\mathbb{N}}$. Then, either $\alpha(\gamma(0))=0$ or $\alpha(\gamma(0)) \neq 0$. If $\alpha(\gamma(0))=0$, then $\bar{\gamma} 1 \in P$. If $\alpha(\gamma(0)) \neq 0$, then $(\exists n \in \mathbb{N}) \beta(n) \neq 0$ implies $(\exists n \in \mathbb{N}) \alpha(n) \neq 0 \wedge(\exists n \in \mathbb{N}) \beta(n) \neq 0$, a contradiction. Thus, $(\forall n \in \mathbb{N}) \beta(n)=0$. Hence, $\bar{\gamma} 0=\langle\rangle \in P$. Therefore, $P$ is a bar.

Define a predicate $Q \subseteq \mathbb{N}^{*}$ by

$$
Q \stackrel{\text { def }}{=} P \cup\{\langle\rangle \mid(\forall n \in \mathbb{N}) \alpha(n)=0\} .
$$

Then, $Q$ is clearly inductive and $P \subseteq Q$. Thus, by $\Pi_{1}^{0}$-BI, we have \langle\rangle$\in Q$, ie

$$
(\forall n \in \mathbb{N}) \alpha(n)=0 \vee(\forall n \in \mathbb{N}) \beta(n)=0
$$

or, equivalently, $\neg(\exists n \in \mathbb{N}) \alpha(n) \neq 0 \vee \neg(\exists n \in \mathbb{N}) \beta(n) \neq 0$.

It is well known that the $\Sigma_{1}^{0}$ bar induction implies LPO (the limited principle of omniscience, also known as the $\Sigma_{1}^{0}$ law of excluded middle); see Troelstra and van Dalen [10, Chapter 4, Exercise 4.8.11]. ${ }^{4}$ Since the continuity axiom WC-N contradicts LLPO (Troelstra and van Dalen [10, Chapter 4, Proposition 6.5]), those results show that the monotonicity of the bar is essential for an intuitionistically acceptable formulation of bar induction. Note that the situation is quite different for the fan theorem; since the $\Pi_{1}^{0}$ fan theorem (the fan theorem with respect to $\Pi_{1}^{0}$ binary bars) is an instance of the full fan theorem, it is intuitionistically acceptable.

\section{Further work}

We now have the following implications.

(1) $\mathrm{BI}_{\mathbf{M}} \Longrightarrow \mathrm{c}-\mathrm{BI} \Longrightarrow \mathrm{BI}_{\mathbf{D}}$.

(2) $\mathrm{BC}-\mathrm{N} \Longrightarrow \mathrm{C}-\mathrm{N} \Longrightarrow \mathrm{PC}-\mathrm{N} \Longrightarrow$ WC-N.

It remains to be seen which of these implications are strict, that is cannot be reversed. In view of the strength of the notion of continuity associated with each principles, we conjecture that all of the above implications are strict.

\footnotetext{
${ }^{4}$ The example of the bar that is used to derive LPO from the $\Sigma_{1}^{0}$ bar induction is attributed to Kleene [8, Section 7.14], but the bar defined in [8, Section 7.14] is not a $\Sigma_{1}^{0}$ set.
} 


\section{Acknowledgements}

We thank Hajime Ishihara and Takako Nemoto for helpful comments on the subject of this paper. The author is supported by Core-to-Core Program A. Advanced Research Networks by Japan Society for the Promotion of Science (JSPS).

\section{References}

[1] M J Beeson, Foundations of Constructive Mathematics, Springer, Berlin, Heidelberg (1985); https://dx.doi.org/10.1007/978-3-642-68952-9

[2] J Berger, The Fan Theorem and Uniform Continuity, from: "New Computational Paradigms. CiE 2005", (S B Cooper, B Löwe, L Torenvliet, editors), Lecture Notes in Comput. Sci. 3526, Springer, Berlin, Heidelberg (2005) 18-22; https://dx.doi.org/10.1007/11494645_3

[3] J Berger, The Logical Strength of the Uniform Continuity Theorem, from: "Logical Approaches to Computational Barriers. CiE 2006", (A Beckmann, U Berger, B Löwe, JV Tucker, editors), Lecture Notes in Comput. Sci. 3988, Springer, Berlin, Heidelberg (2006) 35-39; https://dx.doi.org/10.1007/11780342_4

[4] E Bishop, Foundations of Constructive Analysis, McGraw-Hill, New York (1967)

[5] W A Howard, G Kreisel, Transfinite induction and bar induction of types zero and one, and the role of continuity in intuitionistic analysis, J. Symbolic Logic 31 (1966) 325-358; https://dx.doi.org/10.2307/2270450

[6] H Ishihara, Constructive reverse mathematics: compactness properties, from: "From Sets and Types to Topology and Analysis: Towards Practicable Foundations for Constructive Mathematics", (L Crosilla, P Schuster, editors), Oxford Logic Guides 48, Oxford University Press (2005) 245-267; https://dx.doi.org/10.1093/acprof:oso/9780198566519.003.0016

[7] T Kawai, Formally continuous functions on Baire space, Mathematical Logic Quarterly 64 (2018) 192-200; https://dx.doi.org/10.1002/malq.201700015

[8] S C Kleene, R E Vesley, The foundations of intuitionistic mathematics, especially in relation to recursive functions, North-Holland, Amsterdam (1965)

[9] G Kreisel, A S Troelstra, Formal systems for some branches of intuitionistic analysis, Annals of Mathematical Logic 1 (1970) 229-387;

https://dx.doi.org/10.1016/0003-4843(70)90001-X

[10] A S Troelstra, D van Dalen, Constructivism in Mathematics: An Introduction. Volume I, volume 121 of Studies in Logic and the Foundations of Mathematics, North-Holland, Amsterdam (1988) 
[11] A S Troelstra, D van Dalen, Constructivism in Mathematics: An Introduction. Volume II, volume 123 of Studies in Logic and the Foundations of Mathematics, North-Holland, Amsterdam (1988)

Japan Advanced Institute of Science and Technology

1-1 Asahidai, Nomi, Ishikawa 923-1292, Japan

tatsuji.kawai@jaist.ac.jp

Received: 21 July 2017 Revised: 26 May 2019 